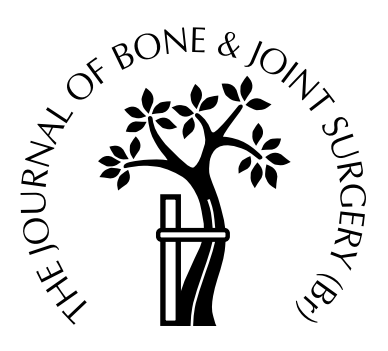

\title{
The depressor function of biceps on the head of the humerus in shoulders with tears of the rotator cuff
}

\author{
Tadato Kido, Eiji Itoi, Norikazu Konno, Akihisa Sano, Masakazu \\ Urayama, Kozo Sato \\ From Akita University School of Medicine, Akita, Japan
}

$\mathbf{W}$ e investigated the function of biceps in 18 patients (19 shoulders) with lesions of the rotator cuff. Their mean age was 59 years. Another series of 18 patients (19 shoulders) with normal rotator cuffs as seen on MRI acted as a control group. Their mean age was 55 years. A brace was used to maintain contraction of biceps during elevation. Anteroposterior radiographs were obtained with the arm elevated at $0^{\circ}, 45^{\circ}$ and $90^{\circ}$ with and without contraction of biceps. The distance between the centre of the head of the humerus and the glenoid was compared in the two groups.

We found that in the group with tears there was significantly greater proximal migration of the head of the humerus at $0^{\circ}$ and $45^{\circ}$ of elevation without contraction of biceps but depression of the head of the humerus at $0^{\circ}, 4^{\circ}$ and $90^{\circ}$ when biceps was functioning. We conclude that biceps is an active depressor of the head of the humerus in shoulders with lesions of the rotator cuff.

J Bone Joint Surg [Br] 2000;82-B:416-9.

Received 13 April 1999; Accepted after revision 18 November 1999

Radiographs of shoulders with tears of the rotator cuff show characteristic changes with cysts in the head of the humerus, ${ }^{1}$ the formation of spurs on the greater tuberosity, the acromion and the glenoid ${ }^{2}$ and superior migration of the head of the humerus. ${ }^{3-8}$ The last occurs as a result of loss of the function of the cuff as a depressor of the head of the humerus and also of the structures which lie between the head of the humerus and the acromion. ${ }^{9}$ Warner and McMahon ${ }^{10}$ have reported superior migration during eleva-

T. Kido, MD, Resident

E. Itoi, MD, Assistant Professor

N. Konno, MD, Resident

A. Sano, MD, Resident

M. Urayama, MD, Resident

K. Sato, MD, Professor and Chairman

Department of Orthopaedic Surgery, Akita University School of Medicine,

1-1-1 Hondo, Akita 010-8543, Japan.

Correspondence should be sent to Dr E. Itoi.

(C2000 British Editorial Society of Bone and Joint Surgery

0301-620X/00/310115 \$2.00

416 tion of the arm in isolated rupture of the long head of biceps in otherwise normal shoulders, which is indirect evidence that biceps acts as a depressor. Our aim was to show directly that active contraction of biceps prevented superior migration of the head of the humerus in shoulders with tears of the rotator cuff.

\section{Patients and Methods}

Between November 1996 and October 1997 we treated surgically 18 patients (19 shoulders) with tears of the rotator cuff which had been diagnosed by arthrography or MRI. There were 14 men and four women with a mean age of 59 years (44 to 77). Eleven shoulders had full-thickness tears, five of which were large $(>3 \mathrm{~cm})$, three medium (1 to $3 \mathrm{~cm})$ and three small $(<1 \mathrm{~cm})$. Eight shoulders had partialthickness tears of which seven were medium and one small. The long head of biceps was intact in all of these patients. A control group of 19 shoulders was asymptomatic with normal rotator cuffs as seen on MRI. There were 15 men and four women with a mean age of 55 years (17 to 77). There was no significant difference in age $(p=0.38)$ and gender $(p=0.99)$ between the two groups.

Anteroposterior radiographs were taken with the arm elevated at $0^{\circ}, 45^{\circ}$ and $90^{\circ}$, the elbow flexed and the forearm supinated, and a brace applied which allowed active contraction of biceps without the other muscles (Fig. 1). The resistant force produced by the band was approximately $1.5 \mathrm{~kg}$. The distance between the centre of the head of the humerus and the glenoid was measured and processed using National Institute of Health software ${ }^{11}$ (NIH Image; National Institute of Health, Bethesda, Maryland) (Fig. 2). In four volunteers with a mean age of 30 years (29 to 31) EMG was performed using the brace and activity in the anterior, middle and posterior deltoid, triceps, trapezius and pectoralis major recorded at $0^{\circ}, 45^{\circ}$ and $90^{\circ}$ of elevation. We were able to confirm that when using the brace, only biceps was contracting significantly. These activities were measured as percentages of the maximum voluntary contraction (\% MVC). ${ }^{12,13}$

Statistical analysis. The results in the two groups were compared using a one-way between-group analysis of variance (ANOVA), with statistical significance set at the 5\% level. 


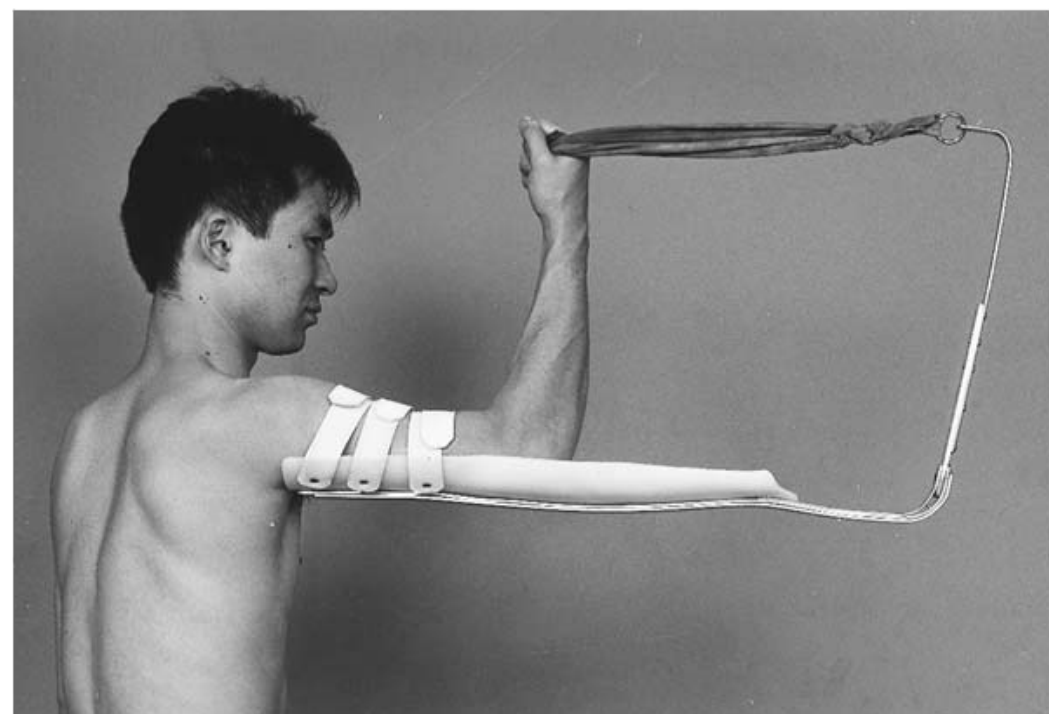

Fig. 1

Arm elevation with a brace. The elbow is flexed at $90^{\circ}$ and the forearm is supinated. The brace is attached to the upper arm and the patient asked to grasp a rubber band, pull it proximally, and keep the elbow flexed against the resistant force.
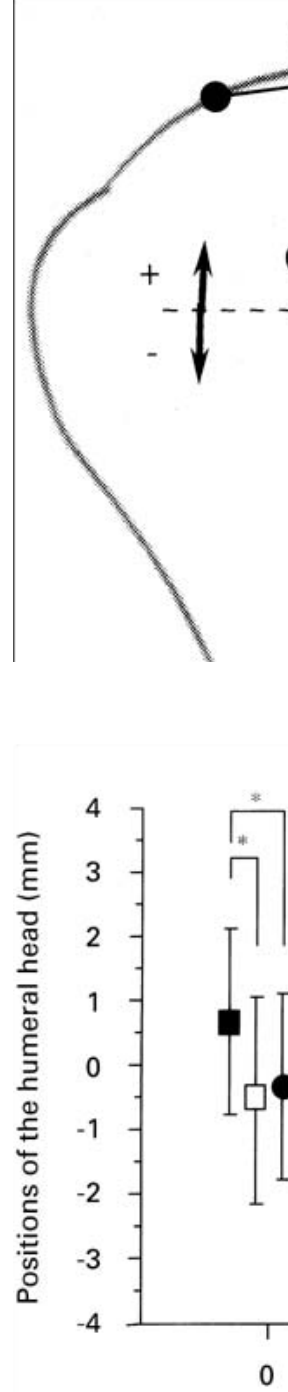

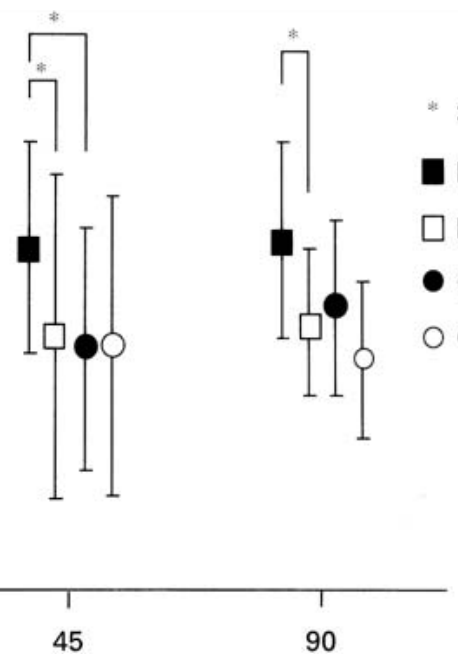

45
$: p<0.05$

RCT BC(-)

$\mathrm{RCT} \mathrm{BC(+)}$

O $\mathrm{CON} \mathrm{BC}(+)$
- $\operatorname{CON} B C(-)$

Fig. 2

Measurement on radiographs. Using the method of Sharkey and Marder ${ }^{11}$ three points arbitrarily chosen on the surface of head of the humerus and wo at the supraglenoid and infraglenoid tubercles were digitised. The centre of the head of the humerus (C) from the former was calculated, and the distance from the perpendicular bisector of the glenoid was measured. The distance (D) was expressed as positive $(+)$ if the centre was above the perpendicular bisector of the glenoid, and negative (-) if it was below the bisector

Fig. 3

The relationship of the position of the head of the humerus to the angle of elevation (degrees). The position of the head of the humerus was significantly higher in the group with tears than in the control group without contraction of biceps at $0^{\circ}(\mathrm{p}=0.0101)$ and at $45^{\circ}$ of elevation $(p=0.0020)$. The head of the humerus was depressed significantly in those with tears with contraction of biceps at $0^{\circ}, 45^{\circ}$ and $90^{\circ} \quad(\mathrm{p}=0.0082$ $\mathrm{p}=0.0259, \mathrm{p}=0.0059$, respectively) $(\mathrm{RCT}$, rotator-cuff tear; CON, control; $\mathrm{BC}(-)$, without contraction of biceps; $\mathrm{BC}(+)$, with contraction of the biceps. 
T. KIDO, E. ITOI, N. KONNO, A. SANO, M. URAYAMA, K. SATO

Table I. Mean ( \pm SD) \% MVC of each muscle with and without biceps loading

\begin{tabular}{|c|c|c|c|c|}
\hline \multirow[b]{2}{*}{ Muscles } & \multirow{2}{*}{$\begin{array}{l}\text { Elevation angle } \\
\text { (degrees) }\end{array}$} & \multicolumn{2}{|l|}{ \%MVC } & \multirow[b]{2}{*}{ p value* } \\
\hline & & Without biceps loading & With biceps loading & \\
\hline Anterior deltoid & $\begin{array}{r}0 \\
45 \\
90\end{array}$ & $\begin{array}{r}6.72 \pm 1.86 \\
8.53 \pm 1.43 \\
14.78 \pm 3.75\end{array}$ & $\begin{array}{l}12.50 \pm 4.41 \\
13.80 \pm 2.63 \\
15.30 \pm 0.92\end{array}$ & $\begin{array}{l}0.1058 \\
0.0387 \\
0.8478\end{array}$ \\
\hline Middle deltoid & $\begin{array}{r}0 \\
45 \\
90\end{array}$ & $\begin{array}{l}6.95 \pm 2.34 \\
8.44 \pm 0.85 \\
7.54 \pm 2.06\end{array}$ & $\begin{array}{r}5.64 \pm 0.92 \\
9.56 \pm 1.83 \\
10.28 \pm 2.64\end{array}$ & $\begin{array}{l}0.4202 \\
0.3913 \\
0.2307\end{array}$ \\
\hline Posterior deltoid & $\begin{array}{r}0 \\
45 \\
90\end{array}$ & $\begin{array}{l}5.02 \pm 3.88 \\
6.38 \pm 3.73 \\
6.47 \pm 4.26\end{array}$ & $\begin{array}{l}5.57 \pm 2.10 \\
5.78 \pm 2.12 \\
8.28 \pm 4.93\end{array}$ & $\begin{array}{l}0.8385 \\
0.8220 \\
0.6544\end{array}$ \\
\hline Biceps & $\begin{array}{r}0 \\
45 \\
90\end{array}$ & $\begin{array}{l}2.78 \pm 0.59 \\
4.71 \pm 0.52 \\
5.24 \pm 1.37\end{array}$ & $\begin{array}{l}12.36 \pm 2.16 \\
10.85 \pm 1.48 \\
12.10 \pm 2.52\end{array}$ & $\begin{array}{l}0.0018 \\
0.0025 \\
0.0143\end{array}$ \\
\hline Triceps & $\begin{array}{r}0 \\
45 \\
90\end{array}$ & $\begin{array}{l}2.04 \pm 0.45 \\
2.66 \pm 0.92 \\
2.29 \pm 0.66\end{array}$ & $\begin{array}{l}3.69 \pm 0.73 \\
3.30 \pm 1.62 \\
3.37 \pm 1.47\end{array}$ & $\begin{array}{l}0.0292 \\
0.5859 \\
0.3135\end{array}$ \\
\hline Trapezius & $\begin{array}{r}0 \\
45 \\
90\end{array}$ & $\begin{array}{l}3.15 \pm 2.08 \\
4.46 \pm 2.72 \\
9.76 \pm 4.54\end{array}$ & $\begin{array}{r}5.36 \pm 1.13 \\
6.49 \pm 1.90 \\
13.30 \pm 6.54\end{array}$ & $\begin{array}{l}0.1812 \\
0.3481 \\
0.4835\end{array}$ \\
\hline Pectoralis major & $\begin{array}{r}0 \\
45 \\
90\end{array}$ & $\begin{array}{l}3.98 \pm 1.67 \\
4.69 \pm 1.68 \\
5.63 \pm 2.06\end{array}$ & $\begin{array}{l}6.31 \pm 0.78 \\
6.83 \pm 0.67 \\
8.85 \pm 0.49\end{array}$ & $\begin{array}{l}0.0934 \\
0.1103 \\
0.0586\end{array}$ \\
\hline
\end{tabular}

* one-way ANOVA

\section{Results}

The position of the head of the humerus was significantly higher in the group with tears of the cuff without contraction of biceps $(p=0.0011)$, and lower with contraction of biceps $(p=0.0012)$ (Fig. 3). EMG showed significant increases in the \% MVC at all angles of elevation only in biceps ( $p<0.0143$; Table I), although there were increases in the activity of anterior deltoid and triceps.

\section{Discussion}

Warner and McMahon ${ }^{10}$ found increased superior migration of the head of the humerus during elevation in patients

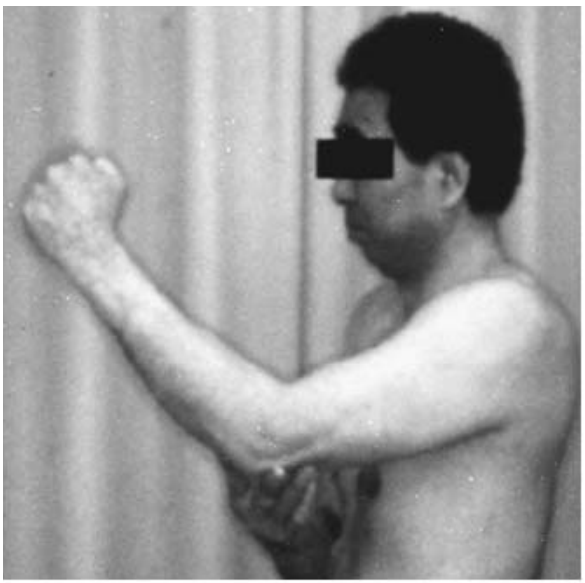

Fig. 4a with rupture of the long head of biceps. Itoi et al ${ }^{14}$ showed that this tendon was wider in cuff-deficient shoulders. Our study has shown that contraction of biceps depressed the head of the humerus both in normal shoulders and those with lesions of the rotator cuff, and that this effect was greater in the group with tears. The movement of the head in cuff-deficient shoulders approached that of normal shoulders after contraction of biceps. This suggests that active contraction of biceps may compensate for the depressor function of the cuff. Kido et $\mathrm{al}^{13}$ showed that EMG activity in biceps increased during elevation in onethird of patients with tears of the cuff. In these patients, biceps may have contracted to stabilise the humeral head. Although pain influences muscle function ${ }^{15}$ it does not

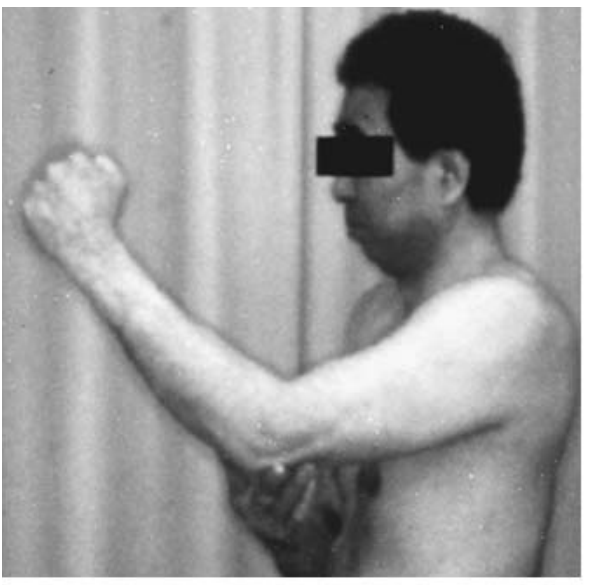

Fig. $4 b$

A 45-year-old carpenter with a massive tear of the rotator cuff of the left shoulder. He could not lift his left arm without biceps loading because of pain (a) but with biceps loading he could do so without pain (b). 
affect superior migration of the head of the humerus during elevation in shoulders with lesions of the cuff. ${ }^{16}$ Although high-resolution MRI has a rate of accuracy of $95 \%$ for the diagnosis of tears of the cuff, ${ }^{17,18}$ it may be difficult to differentiate between complete and incomplete tears. We therefore combined all tears together and were thus not able to relate the effect of the size of the tear to the depressor function of the long head of biceps.

Raised subacromial impingement can only be avoided with adequate depressor function. Payne et $\mathrm{al}^{19}$ reported that subacromial pressure decreased by $10 \%$ after contraction of biceps, and that patients with tears of the cuff may have better function when biceps contracts. Figure 4 illustrates a patient with a large lesion of the cuff who had limited elevation. With a $1 \mathrm{~kg}$ weight in his hand and his elbow flexed, however, functional elevation could be achieved. He was able to work comfortably as a carpenter if he wore a light-weight ( 0.5 to $1 \mathrm{~kg}$ ) wrist band.

The long head of biceps may be damaged or ruptured in association with tears of cuff and in such cases tenotomy or tenodesis has been recommended. ${ }^{20,21}$ On the basis of our findings, however, we would recommend preservation of biceps.

No benefits in any form have been received or will be received from a commercial party related directly or indirectly to the subject of this article.

\section{References}

1. Sano A, Itoi E, Konno N, et al. Cystic changes of the humeral head on MR imaging: relation to age and cuff tears. Acta Orthop Scand 1998;69:397-400.

2. Konno N, Itoi E, Minagawa H, Sato T, Sato K. Cyst formation in the humeral head observed in symptomatic shoulders using MRI. Presented at the 6th International Congress on Surgery of the Shoulder, Helsinki, 1995.

3. Codman EA. The shoulder: rupture of the supraspinatus tendon and other lesions in or about the subacromial bursa. Boston: Thomas Todd, 1934.

4. Golding FC. The shoulder: the forgotten joint. Br J Radiol 1962; 35:149-58
5. Cotton RE, Rideout DF. Tears of the humeral rotator cuff: a radiological and pathological necropsy survey. J Bone Joint Surg [Br] 1964;46-B:314-28.

6. Weiner DS, Macnab I. Superior migration of the humeral head: a radiological aid in the diagnosis of tears of the rotator cuff. $J$ Bone Joint Surg [Br] 1970;52-B:524-7.

7. Kotzen LM. Roentgen diagnosis of rotator cuff tear: report of 48 surgically proven cases. Am J Roentgenol Radium Ther Nucl Med 1971;112:507-11.

8. Poppen NK, Walker PS. Normal and abnormal motion of the shoulder. J Bone Joint Surg [Am] 1976;58-A:195-201.

9. Morrey BF, Itoi E, An K-N. Biomechanics of the shoulder. In: Rockwood CA Jr, Matsen FA III, eds. The shoulder. 2nd ed. Philadelphia: WB Saunders Company, 1998:233-76.

10. Warner JJJP, McMahon PJ. The role of the long head of the biceps brachii in superior stability of the glenohumeral joint. $J$ Bone Joint Surg [Am] 1995;77-A:366-72.

11. Sharkey NA, Marder RA. The rotator cuff opposes superior translation of the humeral head. Am J Sports Med 1995;23:270-5.

12. Kronberg M, Broström L-Å, Németh G. Differences in shoulder muscle activity between patients with generalized joint laxity and normal controls. Clin Orthop 1991;269:181-92.

13. Kido T, Itoi $\mathbf{E}$, Konno $\mathbf{N}$, et al. Electromyographic activities of the biceps during arm elevation in shoulders with rotator-cuff tears. Acta Orthop Scand 1998;69:575-9.

14. Itoi E, Hsu HC, Carmichael SW, Morrey BF, An KN. Morphology of the torn rotator cuff. J Anat 1995;186:429-34.

15. Itoi $\mathbf{E}$, Minagawa $H$, Sato $T$, Sato $K$, Tabata $S$. Isokinetic strength after tears of the supraspinatus tendon. J Bone Joint Surg [Br] 1997;79-B:77-82.

16. Yamaguchi K, Sher JS, Andersen WK, et al. Glenohumeral motion in patients with rotator cuff tears: a comparison of asymptomatic, symptomatic and normal shoulders. 13th Open Meeting, American Shoulder and Elbow Surgeons, San Francisco, February 16, 1997.

17. Itoi E, Kido T, Sano A, Urayama M, Sato K. Which is more useful, the 'full can test' or the 'empty can test' in detecting the torn supraspinatus tendon? Am J Sports Med 1999;27:65-8.

18. Sashi R, Terui M, Narita K, et al. Dual phased array coils for highresolution MRI of the shoulder. Radiat Med 1997;15:13-5.

19. Payne LZ, Deng X-H, Craig EV, Torzilli PA, Warren RF. The combined dynamic and static contributions to subacromial impingement: a biomechanical analysis. Am J Sports Med 1997;25:801-8.

20. Kempf JF, Gleyze P, Bonnomet F, et al. A multicenter study of 210 rotator cuff tears treated by arthroscopic acromioplasty. Arthroscopy 1999;15:56-66.

21. Walch G, Madonia G, Pozzi I, Riand N, Levigne C. Arthroscopic tenotomy of the long head of the biceps in rotator cuff ruptures. In: Gazielly DF, Gleyze P, Thomas T, eds. The cuff. Paris: Elsevier, 1997:350-5. 